\title{
A genome-wide association scan implicates DCHS2, RUNX2, GLI3, PAX1 and EDAR in human facial variation
}

Kaustubh Adhikari ${ }^{1, \star}$, Macarena Fuentes-Guajardo ${ }^{1,2, \star}$, Mirsha Quinto-Sánchez ${ }^{3}$, Javier Mendoza-Revilla,4, Juan Camilo Chacón-Duque ${ }^{1}$, Victor Acuña-Alonzo, ${ }^{1,5}$, Claudia Jaramillo6, William Arias ${ }^{6}$, Rodrigo Barquera Lozano ${ }^{5,7}$, Gastón Macín Pérez ${ }^{5,7}$, Jorge Gómez-Valdés ${ }^{8}$, Hugo Villamil-Ramírez ${ }^{7}$, Tábita Hunemeier ${ }^{9} \uparrow$, Virginia Ramallo ${ }^{3,9}$, Caio C. Silva de Cerqueira ${ }^{3,9}$, Malena Hurtado ${ }^{4}$, Valeria Villegas ${ }^{4}$, Vanessa Granja ${ }^{4}$, Carla Gallo ${ }^{4}$, Giovanni Poletti ${ }^{4}$, Lavinia Schuler-Faccini ${ }^{9}$, Francisco M. Salzano ${ }^{9}$, Maria- Cátira Bortolini ${ }^{9}$, Samuel Canizales-Quinteros ${ }^{7}$, Michael Cheeseman ${ }^{10}$, Javier Rosique ${ }^{11}$, Gabriel Bedoya ${ }^{6}$, Francisco Rothhammer ${ }^{12}$, Denis Headon ${ }^{10}$, Rolando González-José ${ }^{3}$, David Balding ${ }^{1,13}$ \& Andrés Ruiz-Linares ${ }^{1}$

We report a genome-wide association scan for facial features in $\sim 6,000$ Latin Americans. We evaluated 14 traits on an ordinal scale and found significant association $\left(P\right.$ values $\left.<5 \times 10^{-8}\right)$ at single-nucleotide polymorphisms (SNPs) in four genomic regions for three nose-related traits: columella inclination (4q31), nose bridge breadth (6p21) and nose wing breadth (7p13 and 20p11). In a subsample of $\sim 3,000$ individuals we obtained quantitative traits related to 9 of the ordinal phenotypes and, also, a measure of nasion position. Quantitative analyses confirmed the ordinal-based associations, identified SNPs in 2q12 associated to chin protrusion, and replicated the reported association of nasion position with SNPs in PAX3. Strongest association in 2q12, 4q31, 6p21 and 7p13 was observed for SNPs in the EDAR, DCHS2, RUNX2 and GLI3 genes, respectively. Associated SNPs in 20 p11 extend to $P A X 1$. Consistent with the effect of EDAR on chin protrusion, we documented alterations of mandible length in mice with modified Edar funtion.

\footnotetext{
${ }^{1}$ Department of Genetics, Evolution and Environment, UCL Genetics Institute, University College London, London WC1E 6BT, UK. ${ }^{2}$ Departamento de Tecnología Médica, Facultad de Ciencias de la Salud, Universidad de Tarapacá, Arica 1000009, Chile. ${ }^{3}$ Centro Nacional Patagónico, CONICET, Unidad de Diversidad, Sistematica y Evolucion, Puerto Madryn U912OACD, Argentina. ${ }^{4}$ Laboratorios de Investigación y Desarrollo, Facultad de Ciencias y Filosofía, Universidad Peruana Cayetano Heredia, Lima 31, Perú. ${ }^{5}$ Laboratorio de Genética Molecular, Escuela Nacional de Antropologia e Historia, México City 14030 , México. ${ }^{6}$ GENMOL (Genética Molecular), Universidad de Antioquia, Medellín 5001000, Colombia. ${ }^{7}$ Unidad de Genómica de Poblaciones Aplicada a la Salud, Facultad de Química, UNAM-Instituto Nacional de Medicina Genómica, México City 4510, México. ${ }^{8}$ Departamento de Anatomía, Facultad de Medicina, Universidad Nacional Autónoma de México (UNAM), México City 04510, México. ${ }^{9}$ Departamento de Genética, Universidade Federal do Rio Grande do Sul, Porto Alegre 91501-970, Brasil. ${ }^{10}$ Division of Developmental Biology, The Roslin Institute and Royal (Dick) School of Veterinary Studies, University of Edinburgh, Midlothian EH25 9RG, UK. ${ }^{11}$ Departamento de Antropología, Universidad de Antioquia, Medellín 5001000, Colombia. ${ }^{12}$ Instituto de Alta Investigación, Universidad de Tarapacá, Arica 1000000, Chile. ${ }^{13}$ Schools of BioSciences and Mathematics and Statistics, University of Melbourne, Melbourne, Victoria 3010, Australia. * These authors contributed equally to this work. † Present address: Departamento de Genética e Biologia Evolutiva, Universidade de São Paulo, São Paulo 05508-090, Brasil. Correspondence and requests for materials should be addressed to A.R.-L. (email: a.ruizlin@ucl.ac.uk).
} 
$\mathrm{H}$ umans show extensive variation in facial features, physical anthropologists having long used this variation to examine human population diversification, including the possibility that these features have been influenced by adaptation to the environment ${ }^{1-3}$. It has also been proposed that the diversity of human faces could have evolved partly to facilitate individual recognition, a key aspect of social interaction ${ }^{4}$. Other than their considerable evolutionary interest, a range of categorical and quantitative craniofacial traits have been used in forensics for the purpose of human identification and estimation of ancestry ${ }^{5,6}$. Consistent with facial variation being under strong genetic control, heritabilities of $\sim 60-90 \%$ have been estimated for facial phenotypes ${ }^{7-9}$. The characterization of gene mutations in patients with dysmorphologies and in animal models has enabled the identification of rare genetic variants with major effects on facial development ${ }^{10}$. However, our current understanding of the molecular genetic basis of variable face appearance in the general human population is scant. Only two genome-wide association studies (GWAS) for facial features have so far been published ${ }^{9,11}$. These were carried out in Europeans and only one gene region (PAX3) was consistently associated with a facial feature in both studies (position of the nasion, the deepest point on the nasal bridge $)^{9,11}$.

We recently reported the successful identification of genes influencing outer ear (pinna) morphology using a three-point ordinal phenotyping approach ${ }^{12}$. Here, we extend this methodology to other aspects of facial variation. In addition, in a subsample of individuals, we obtained quantitative measures related to the ordinal phenotypes examined. Our analyses allowed us to replicate the reported $P A X 3 /$ nasion position association and to identify five other gene regions impacting on face (mostly nose) morphology (EDAR, DCHS2, RUNX2, GLI3 and PAX1). These genes are known to play important roles in craniofacial development.

\section{Results}

Study sample and ordinal phenotypes. Our study sample is part of the CANDELA cohort collected in Latin America ${ }^{13}$ (Supplementary Table 1). Using facial photographs of 6,275 individuals, we assessed 14 facial features on an ordered categorical scale reflecting the distinctiveness of each trait (Fig. 1, Supplementary Table 2). We included features of the lower face: chin shape, chin protrusion and upper/lower lip thickness; the middle face: cheekbone protrusion, breadth of nasal root, bridge and wing, columella inclination, nose protrusion, nose profile and nose tip shape; and the upper face: brow-ridge protrusion and forehead profile. These features were selected based on their documented variation in Europeans ${ }^{5}$. We found them to be reliably scored (Supplementary Table 3) and to also show extensive variation in the CANDELA sample (Supplementary Fig. 1). Individuals were genotyped on Illumina's OmniExpress BeadChip and imputation performed using 1000 Genomes data. After quality-control filters, final analyses were carried out on 671,038 genotyped single-nucleotide polymorphisms (SNPs) and 9,117,642 imputed SNPs in 5,958 individuals. On the basis of the genome-wide SNP data, average autosomal admixture proportions for the full sample were estimated as: 50\% European, 45\% Native American and 5\% African (Supplementary Fig. 2).

Significant correlations were observed between the ordinal phenotypes (using a Bonferroni-adjusted permutation $P$ value threshold for significance of $6 \times 10^{-4}$, Supplementary Table 4A). Strongest correlation was observed between upper and lower lip thickness $(r=0.72)$, followed by forehead profile and brow ridge protrusion $(r=0.57)$. The three traits related to nose width (root, bridge and wing breadth) show positive correlations among them $(r=0.16-0.37)$ and negative correlations with nose protrusion $(r=-0.08$ to -0.25$)$. Several of the facial traits examined also show moderate (and significant) correlations with age, sex, body mass index (BMI) and genetic ancestry (Supplementary Table 4B). The strongest correlation with sex was seen for brow ridge protrusion and forehead profile $(r=-0.62$ and $r=-0.47$, respectively). Age correlates most strongly with upper and lower lip thickness ( $r=-0.19$ and $r=-0.24$, respectively), while the strongest correlation for BMI was seen with brow-ridge protrusion $(r=0.17)$. Genetic ancestry has strongest correlation with lip thickness (European ancestry being negatively correlated with upper and lower lip thickness, $r=-0.25$ and $r=-0.16$, respectively). European ancestry is also significantly correlated with all the nose features examined, particularly with nose protrusion $(r=0.18)$ and nose wing breadth $(r=-0.15)$. On the basis of a kinship matrix derived from the SNP data ${ }^{14}$, we estimated narrow-sense heritability for the facial traits using GCTA $^{15}$. We found moderate (and significant) values for all traits, with the highest heritability being estimated for nose protrusion (0.47) and the lowest for columella inclination (0.20; Supplementary Table 5). Similar (or higher) heritabilities have been estimated for a range of facial traits using family data ${ }^{7,8,16}$.

GWAS for ordinal phenotypes. We performed genome-wide association tests using multivariate linear regression, as implemented in PLINK ${ }^{17}$, using an additive genetic model adjusting for: age, sex, BMI and the first five principal components (PCs, Supplementary Fig. 3) computed from the SNP data. The resulting statistics showed no evidence of residual population stratification for any of the traits (Supplementary Fig. 3). Three of the nose traits examined (columella inclination, nose bridge and wing breadth) showed genome-wide significant association $\left(P\right.$ values $\left.<5 \times 10^{-8}\right)$ with SNPs in at least one genomic region (Fig. 1, Table 1). Columella inclination and nose bridge breadth show association with SNPs in a single region $(4 \mathrm{q} 31$ and $6 \mathrm{p} 21$, respectively), while nose wing breadth shows association with SNPs in two genomic regions (7p13 and 20p11). To account for the multiple phenotypes tested, we performed a global falsediscovery rate test across all traits and SNPs and identified the same significantly associated regions (Supplementary Table 6). We examined association for each index SNP (the variant with the lowest $P$ value in a chromosomal region; Table 1) in all countries sampled separately and combined results as a meta-analysis using METAL (Supplementary Table 7) (ref. 18). For all associations, significant effects were in the same direction in all countries, the variability of effect size across countries reflecting sample size (Fig. 2). There was no significant effect size heterogeneity across countries for any of the associations. To exploit the correlations observed between various facial traits, we performed a multivariate $\mathrm{GWAS}^{19}$, but this approach did not identify any additional associated regions (Supplementary Table 8).

Follow-up analyses. Subsequent to the GWAS described above, we obtained data from an additional set of 501 individuals from the same countries as for the GWAS and used this as a replication sample (descriptive features of this sample are presented in Supplementary Fig. 4). These individuals were phenotyped and genotyped as for the GWAS sample. Association tests for the four index SNPs in Table 1 were performed using the same regression model as for the GWAS, with a Bonferroni-adjusted threshold for significance of $0.05 / 4=0.0125$. All tests were found to be significant in this replication sample (Table 1).

We also followed-up the ordinal facial trait GWAS by obtaining facial measurements (distances and angles) related to 

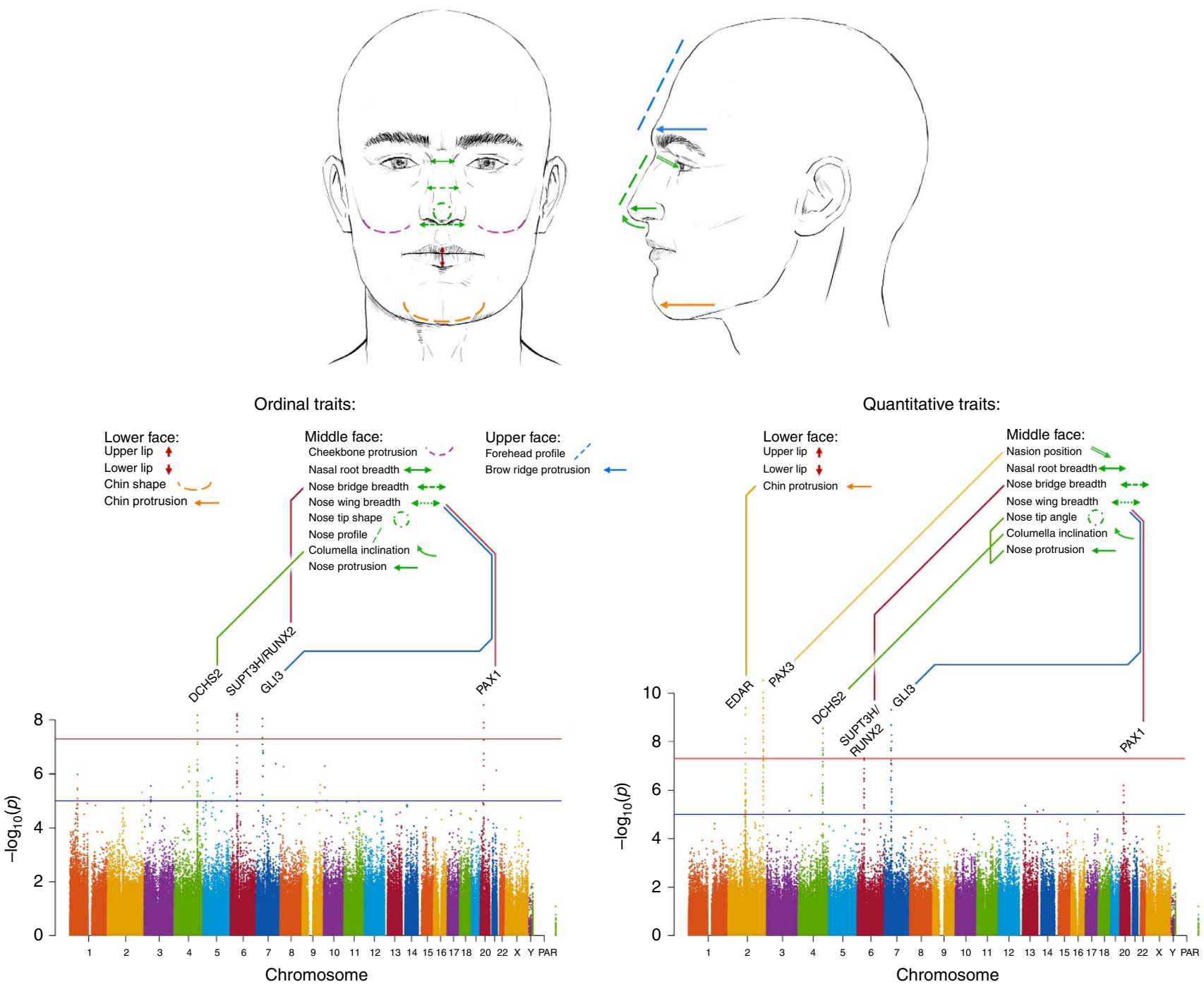

Figure 1 | Overview of GWAS for facial features in the CANDELA sample. We first carried out a GWAS using data for 14 ordinal facial features from the lower, middle and upper face in 5,958 individuals. For follow-up, we obtained quantitative proxies for 9 of the 14 ordinal traits initially examined (and also obtained a measure of nasion position) in a subset of 2,955 individuals, and performed another GWAS. For convenience, we summarize results across traits on a single 'composite' Manhattan plot shown at the bottom of the figure (ordinal traits on the left and quantitative traits on the right). Each Manhattan plot displays all the SNPs with $P$ values exceeding thresholds for genome-wide suggestive $\left(10^{-5}\right.$, blue line) or genome-wide significance $\left(5 \times 10^{-8}\right.$, red line) for any trait. To avoid cluttering the figure, $P$ values not reaching the suggestive threshold (that is, whose significance can be disregarded) are shown only for one trait (upper lip thickness). The names of the candidate genes closest to each association peak are provided (Table 1). These genes are connected with the list of associated facial features via lines of different colour. The location of these features is illustrated on the face drawings shown at the top of the figure. Face drawings were prepared by Emiliano Bellini. PAR, pseudo-autosomal region.

Table 1 | Properties of index SNPs in chromosomal regions showing genome-wide significant association to ordinal facial traits.

\begin{tabular}{|c|c|c|c|c|c|c|c|c|}
\hline $4 q 31$ & rs12644248 & $\begin{array}{l}\text { Columella } \\
\text { inclination }\end{array}$ & $7 \times 10^{-9}$ & DCHS2 & $A>G$ & $-8.40 \times 10^{-2}$ & 0.49 & $4 \times 10^{-3}$ \\
\hline $6 \mathrm{p} 21$ & rs1852985 & $\begin{array}{l}\text { Nose bridge } \\
\text { breadth }\end{array}$ & $6 \times 10^{-10}$ & SUPT3H/RUNX2 & $\mathrm{C}>\mathrm{T}$ & $6.90 \times 10^{-2}$ & 0.71 & $5 \times 10^{-3}$ \\
\hline 7p13 & rs17640804 & $\begin{array}{l}\text { Nose wing } \\
\text { breadth }\end{array}$ & $9 \times 10^{-9}$ & GLI3 & $C>T$ & $-6.50 \times 10^{-2}$ & 0.62 & $6 \times 10^{-3}$ \\
\hline
\end{tabular}



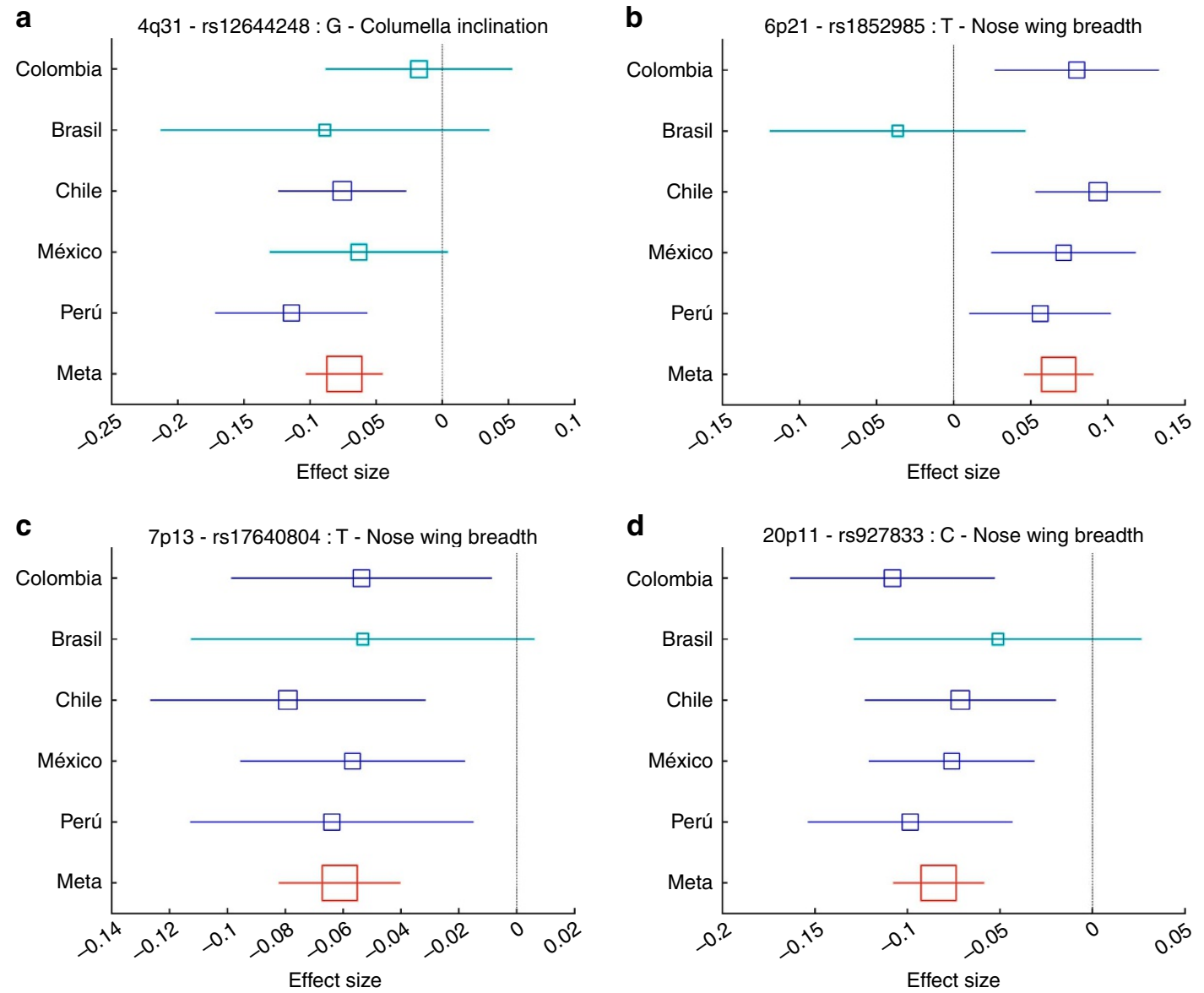

Figure 2 | Effect sizes (regression coefficients) for the derived allele at index SNPs in the genome regions associated with ordinal face traits. (a) 4q31 rs12644248, (b) 6p21 rs1852985, (c) 7 p13 rs17640804, (d) 20 p11 rs927833. Estimates obtained in each country are shown as blue boxes. Red boxes indicate estimates obtained in the meta-analysis. Box size is proportional to sample size. Horizontal bars indicate confidence intervals representing $2 \times$ standard errors. Intervals that include zero (that is, non-significant effects) are shown in light blue.

the ordinal traits initially examined and performing a GWAS on these quantitative data. These measurements were obtained mainly using three-dimensional (3D) anatomical landmark coordinates available for 2,955 of the individuals included in the ordinal trait GWAS ${ }^{20}$ (Supplementary Fig. 5a). These landmarks allowed us to define quantitative proxies for seven of the ordinal facial traits, the other traits having no appropriate 3D landmarks allowing related measurements to be obtained (Supplementary Table 9). Since the ordinal assessment of nose root and bridge breadth produced genome-wide significant associations (but could not be measured with the 3D landmarks available), we carried out 2D landmarking of the frontal photographs of these 2,955 individuals and also obtained measurements for these two traits (Supplementary Table 10, Supplementary Fig. 5b). In addition, we used the 3D landmark coordinates to obtain a measure of nasion position so as to evaluate in our sample the reported association of this feature with SNPs in the PAX3 gene region ${ }^{9,11}$.

The ordinal variables showed a moderate-to-high (and significant) correlation with the quantitative variables (all permutation $P$ values $<0.0005$; Supplementary Table 11 and Supplementary Fig. 6). Correlation between ordinal and quantitative traits was strongest for nose wing breadth and lower lip thickness (both with $r=0.70$ ) and lowest for columella inclination $(r=0.16)$. The pattern of correlation among quantitative traits was similar to that observed for the ordinal traits, as was the correlation between quantitative traits and covariates (Supplementary Table 12). As expected for continuous variables, heritability estimates based on the quantitative phenotypes (Supplementary Table 13) are higher than obtained for the ordinal phenotypes and more in line with published estimates $7,8,16$.

As before, we performed a GWAS for the quantitative traits using an additive multivariate regression model adjusting for age, sex, BMI and the first five PCs. We replicated the reported association of nasion position with SNPs in 2q35 overlapping the $P A X 3$ gene region, with strongest association seen for rs7559271 ( $P$ value of $4 \times 10^{-11}$, Fig. 1, Table 2, Supplementary Fig. 7a). This is the same SNP producing strongest association in the Paternoster et al. ${ }^{11}$ GWAS. In addition, we observed genomewide significant association for six of the nine quantitative proxies of the ordinal traits initially examined (Fig. 1, Table 2). As for the ordinal assessments, the quantitative analysis of columella inclination, nose bridge breadth and nose wing breadth produced genome-wide significant associations with SNPs in 4q31, 6p21 and 7 p13, respectively (Fig. 1, Tables 1 and 2). In addition, the $4 \mathrm{q} 31$ region also showed genome-wide significant association to two other measurements related to nose morphology: nose protrusion and nose tip angle, with strongest $P$ values for SNPs rs2045323 of $1 \times 10^{-8}$ and $2 \times 10^{-8}$, respectively. SNPs in $4 \mathrm{q} 31$ produced small but not genome-wide significant $P$ values in the ordinal assessment of nose protrusion and nose tip angle (strongest $P$ values of $4 \times 10^{-4}$ and $3 \times 10^{-4}$, respectively). The 20 p11 region, showing genome-wide significant association in the 
Table 2 | Properties of index SNPs in regions showing genome-wide significant association to quantitative facial traits.

\begin{tabular}{|c|c|c|c|c|c|c|c|}
\hline $\begin{array}{l}\text { Chromosomal } \\
\text { region }\end{array}$ & Index SNP & Associated trait & P-value & 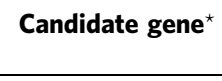 & Alleles $^{\dagger}$ & Effect size & $\begin{array}{c}\text { Percentage of variance } \\
\text { explained }\end{array}$ \\
\hline $2 q 12$ & rs3827760 & Chin protrusion & $4 \times 10^{-10}$ & EDAR & $A>G$ & $-7.60 \times 10^{-3}$ & 1.32 \\
\hline $2 q 35$ & rs7559271 & Nasion position & $4 \times 10^{-11}$ & PAX3 & $A>G$ & $8.20 \times 10^{-2}$ & 1.33 \\
\hline $4 q 31$ & rs2045323 & Columella inclination & $3 \times 10^{-9}$ & DCHS2 & $G>A$ & $1.80 \times 10^{-2}$ & 0.63 \\
\hline $4 q 31$ & rs2045323 & Nose protrusion & $1 \times 10^{-8}$ & DCHS2 & $G>A$ & $-5.90 \times 10^{-4}$ & 0.95 \\
\hline $4 q 31$ & rs2045323 & Nose tip angle & $2 \times 10^{-8}$ & DCHS2 & $G>A$ & $1.60 \times 10^{-2}$ & 1.08 \\
\hline $6 p 21$ & rs1852985 & Nose bridge breadth & $2 \times 10^{-8}$ & SUPT3H/RUNX2 & $\mathrm{C}>\mathrm{T}$ & $4.40 \times 10^{-4}$ & 1.18 \\
\hline $7 \mathrm{p} 13$ & rs17640804 & Nose wing breadth & $5 \times 10^{-10}$ & GLI3 & $\mathrm{C}>\mathrm{T}$ & $-4.90 \times 10^{-4}$ & 1.15 \\
\hline
\end{tabular}

SNP, single-nucleotide polymorphism.

*For intragenic SNPs, gene names are shown in bold.

$\dagger$ Derived alleles are shown after ancestral alleles.

$\ddagger$ Columella inclination was measured as an angle which decreases at greater ordinal columella inclination (Supplementary Table 9, Supplementary Fig. 6f). Therefore, the allelic effects for the quantitative and ordinal assessments of this trait (Table 1) are of opposite sign. rs12644248, the index SNP associated with categorical columella inclination has a $P$ value of $4 \times 10^{-8}$ for association with the quantitative assessment of columella inclination.

ordinal assessment of nose wing breadth, showed genome-wide suggestive association in the quantitative trait GWAS (strongest $P$ value of $6 \times 10^{-7}$ for SNP rs927833). Other than reproducing the associations detected with ordinal traits, the quantitative analyses detected a genome-wide significant association to chin protrusion for markers in $2 \mathrm{q} 12$ (strongest $P$ value of $4 \times 10^{-10}$, for rs3827760; Fig. 1 and Table 2). This marker had an association $P$ value of $1 \times 10^{-4}$ in the ordinal assessment of chin protrusion.

A regression model similar to the one used in the GWAS analyses explains up to $\sim 30 \%$ of the phenotypic variation for the traits with significant SNP associations, with each of the associated SNPs explaining about $1 \%$ of variation in the trait (Tables 1 and 2, Supplementary Table 14). The estimates of trait variance explained by associated SNPs are similar to those calculated for other anthropometric traits and are very close to the estimates obtained in a previous GWAS for facial features ${ }^{11}$.

To assess independent evidence of association for the regions implicated here, we examined SNPs that produced at least genome-wide suggestive $P$ values in the two GWAS for facial features that have been published ${ }^{9,11}$. We found that SNP rs2108166, $5.5 \mathrm{~kb}$ from and in high $\mathrm{LD}\left(r^{2}=0.77, \mathrm{D}^{\prime}=1\right)$ with the index SNP of the 7p13 region we found associated with nose wing breadth (rs17640804), produced an association $P$ value of $5 \times 10^{-7}$ with the same trait in the study of Liu et al. ${ }^{9}$ In addition, evidence of association between rs3827760 and chin shape has recently been reported in a candidate gene study of a Central Asian population ${ }^{21}$.

It has been suggested that gene regions associated with nonsyndromic cleft lip and palate (NSCL/P) might impact on normal variation in facial morphology 9,22 . Although the regions reported to be associated with NSCL/P do not overlap with those identified here, we selected index SNPs in each NSCL/P region and tested for association of these SNPs with the facial traits that we examined (Supplementary Table 15). Few tests survived Bonferroni correction, mostly involving SNPs associated with quantitative nose-breadth traits (nose root, nose bridge and nose wing breadth; Supplementary Table 15A). A global one-sided Kolmogorov-Smirnoff test was significant both for ordinal and quantitative traits ( $P$ value $\sim 10^{-3}$; Supplementary Table 15B) and a polygenic risk score test combining all 15 index SNPs was significant for the nose-breadth traits (Supplementary Table 15C). A more precise evaluation of the impact of NSCL/P-associated variants on facial variation in the general population requires further investigation.

Candidate genes in regions associated with facial morphology. SNPs in 2 q12 associated with chin protrusion show extensive LD and overlap the $3^{\prime}$-half of the EctodysplasinA (EDA) receptor gene (EDAR; Fig. 3a). The derived G allele at the index SNP in this region (rs3827760) encodes a functional substitution in the intracellular death domain of EDAR (370A) and is associated with reduced chin protrusion (Table 2). EDAR is part of the EDA signalling pathway (comprising EDA, EDAR and EDARADD (the EDAR-binding death domain adaptor protein)) which specifies prenatally the location, size and shape of ectodermal appendages (such as hair follicles, teeth and glands) ${ }^{23}$. The death domain has been shown to be involved in the interaction of EDAR with EDARADD, the 370A form having higher activity than the ancestral variant ${ }^{24}$. The $G$ allele at rs3827760 is not present in Europeans and Africans but is seen at high frequency in East Asians and is essentially fixed in Native Americans (Table 3). This SNP has been associated in East Asians with characteristic tooth morphologies, hair type and sweat gland density ${ }^{25-27}$. Recently, we showed, in the same study sample examined here, that rs3827760 impacts on aspects of pinna morphology, including: lobe size and attachment, ear protrusion and helix rolling ${ }^{12}$. Mutations in the EDA pathway cause hypohidrotic ectodermal dysplasia ${ }^{28}$. This disorder is characterized by a reduced number of sweat glands, oligodontia, decrease in the amount of hair and facial dysmorphia, including a markedly protrusive $\operatorname{chin}^{29}$.

Mouse Edar mutant and transgenic lines with either abolished or increased expression of Edar have been described and these mice show features related to several of the phenotypes associated with $E D A R$ in humans s $^{12,30,31}$. Of particular interest, we recently documented that these mice show changes in ear morphology consistent with the effects of EDAR on human ear shape variation $^{12}$. We therefore compared mandible length in Edar wild-type mice with Edar ${ }^{\text {dlJ }}$ and EdarTg951 mutant mice (Supplementary Figs 8 and 9), which have a loss and a gain of Edar function, respectively ${ }^{31,32}$. We found a significant association of mandible length with genotype, with the length decreasing at greater Edar function, consistent with the association of the $370 \mathrm{~A}$ variant with decreased chin protrusion detected in the CANDELA sample (Fig. 4, Supplementary Table 16). Consistent with the mandible length changes we detect in Edar mutant lines, it has been reported that Eda mouse mutants also show mandibular morphology alterations ${ }^{33}$. The impact of the Eda pathway on mandibular morphology has been interpreted as resulting from epithelial-mesenchymal interactions during mouse craniofacial development ${ }^{33}$.

SNPs in the $4 \mathrm{q} 31$ region with $P$ values above the suggestive association threshold in the ordinal trait assessment of columella inclination extend over $\sim 400 \mathrm{~kb}$ from the $3^{\prime}$-half of the Dachsous Cadherin-Related 2 gene (DCHS2) into the DCHS2-SFRP2 
a

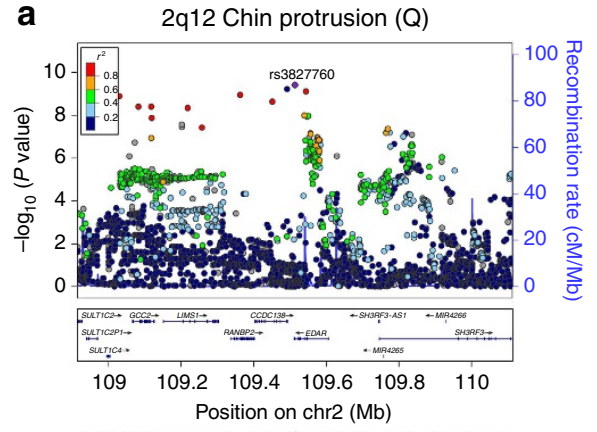

b

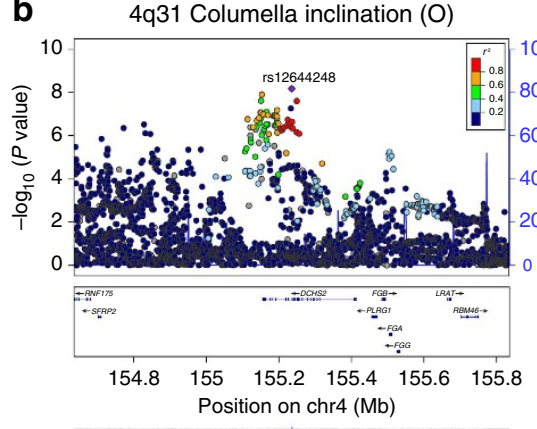

C

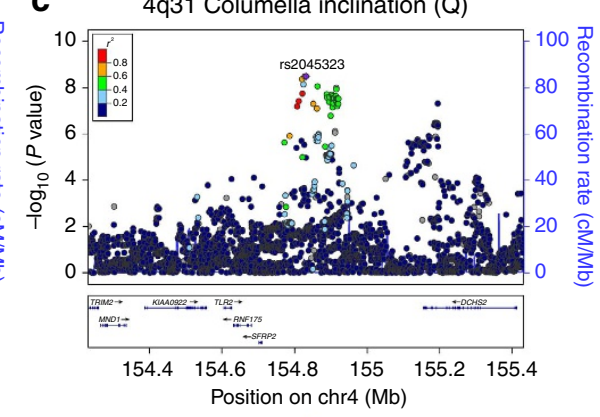

d

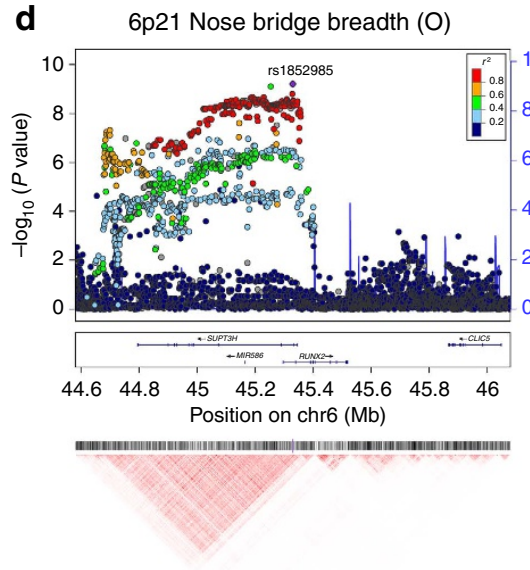

e

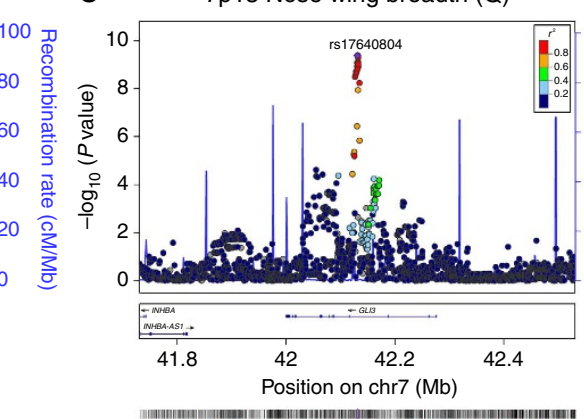

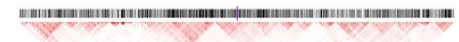

Figure 3 | Genomic regions showing genome-wide significant association to face traits. For each facial feature we show the results that achieved strongest statistical significance regardless of the type of variable analysed (ordinal, O; or quantitative, Q). (a) 2q12 (Q), (b) 4q31 (O), (c) 4q31 (Q), (d) 6p21 (O), (e) 7p13(Q), (f) 20p11 (O). Plots not shown here are shown in Supplementary Fig. 7. Association results (on a - log 10 P scale; left $y$-axis) are shown for SNPs $\sim 500 \mathrm{~kb}$ on either side of the index SNP (purple diamond; Table 1) with the marker (dot) colour indicating the strength of LD ( $r^{2}$ ) between the index SNP and that SNP in the 1000 genomes AMR data set. Local recombination rate in the AMR data is shown as a continuous blue line (scale on the right $y$-axis). Genes in each region, their intron-exon structure, direction of transcription and genomic coordinates (in Mb, using the NCBI human genome sequence, Build 37, as reference) are shown at the bottom. Plots were produced with LocusZoom ${ }^{68}$. Below each region we also show an LD heatmap (using $r^{2}$, ranging from red indicating $r^{2}=1$ to white indicating $r^{2}=0$ ) produced using a MATLAB ${ }^{59}$ implementation similar to Haploview ${ }^{69}$.

Table 3 | Population frequency of derived alleles at index SNPs associated with facial features in the CANDELA sample.

\begin{tabular}{|c|c|c|c|c|c|c|c|}
\hline $2 q 12$ & rs3827760 & G & 0 & 0 & 94 & 98 & 42 \\
\hline $4 q 31$ & rs12644248 & G & 0 & 2 & 15 & 46 & 26 \\
\hline $4 q 31$ & rs2045323 & $A$ & 9 & 3 & 18 & 66 & 34 \\
\hline $6 p 21$ & rs1852985 & $\mathrm{T}$ & 13 & 20 & 24 & 55 & 30 \\
\hline 7p13 & rs17640804 & $\mathrm{T}$ & 72 & 82 & 96 & 36 & 61 \\
\hline
\end{tabular}

SNP, single-nucleotide polymorphism.

${ }^{*} \mathrm{CEU}, \mathrm{YRI}, \mathrm{CHB}$ are Europeans, Yoruba and Chinese from the 1000 genomes project. NAM are Native Americans and CAN is the CANDELA sample examined here. NAM data are from populations included in Reich et al. ${ }^{70}$ 


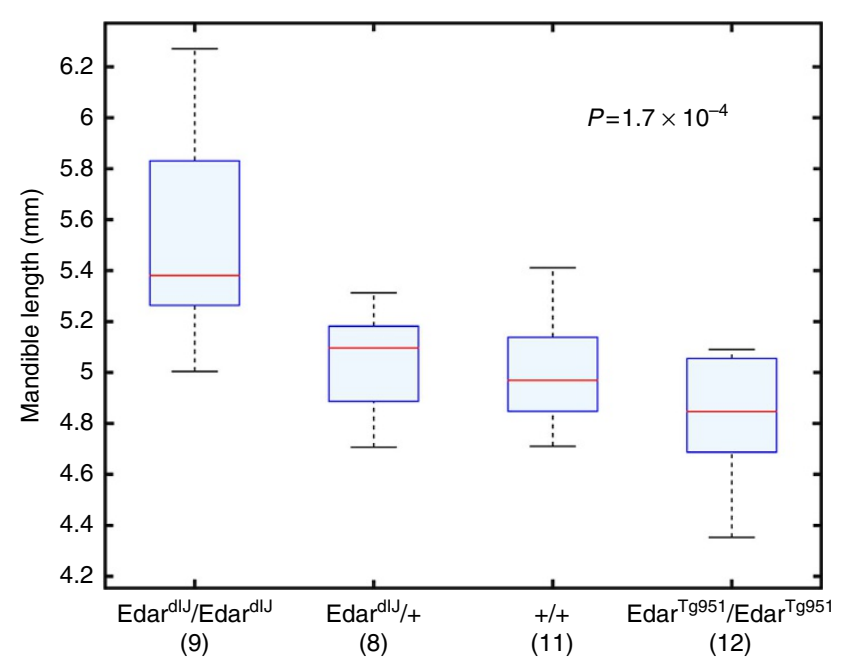

Figure 4 | Effect of Edar genotype on mouse mandible length. We show boxplots of mandible length ( $y$-axis) in mice with different Edar genotypes ( $x$-axis). The measure of mandible length shown is the projected distance between head landmarks 5 and 10 (Supplementary Figs 8 and 9).

Regression analysis indicates a significant effect of Edar genotype on mandible length ( $P$ value $1.7 \times 10^{-4}$ ). Significant results were also obtained for other measurements of mandible length (Supplementary Table 16). Boxplot whiskers extend to data points within 1.5 times the interquartile range on both sides. The numbers in parenthesis below genotypic categories refer to the number of mice examined for each genotype.

(Secreted Frizzled-related protein 2) intergenic region (Fig. 3b), with strongest association seen for SNP rs12644248 within DCHS2 $\left(P\right.$ value $\left.7 \times 10^{-9}\right)$. Noticeably, although association analyses based on the quantitative assessment of columella inclination also show genome-wide significant association for rs12644248 ( $P$ value of $4 \times 10^{-8}$ ), the quantitative analyses show that SNPs in the DCHS2-SFRP2 intergenic region have an even stronger association, peaking at rs2045323 ( $P$ value of $3 \times 10^{-9}$, Table 2, Fig. 3c). A similar pattern of association is seen for the quantitative assessments of nose protrusion and nose tip angle, with strongest association for both traits being observed for rs2045323 ( $P$ values of $1 \times 10^{-8}$ and $2 \times 10^{-8}$, respectively, Table 2, Supplementary Fig. 7), association with rs 12644248 only exceeding the genome-wide suggestive threshold $(P$ values of $8 \times 10^{-6}$ and of $6 \times 10^{-6}$ for nose protrusion and nose tip angle, respectively). SNP rs2045323 is not in strong LD with rs 12644248 and tests conditioned on either SNP attenuate the signal of association at the other SNP but do not abolish it entirely (Supplementary Fig. 10). These observations suggest that the signal of association around rs2045323 in the DCHS2-SFRP2 intergenic region is somewhat independent from that peaking at rs12644248 within DCHS2. Intergenic SNP rs2045323 is located in an evolutionarily conserved region (Supplementary Fig. 11), suggesting that this SNP could play a role in the regulation of genes in the region. DCHS2 is a calcium-dependent cell-adhesion protein which has recently been shown to participate in a regulatory network controlling cartilage differentiation and polarity during vertebrate craniofacial development ${ }^{34}$. This network includes SOX9, a well-known regulator of cartilage differentiation, mutations of which lead in humans to Campomelic Dysplasia (OMIM \#114290) a disorder characterized by a range of craniofacial defects. Although DCHS2 seems the strongest candidate in the $4 \mathrm{q} 31$ region, SFRP2 is also an interesting candidate, in that it has been shown that this gene is expressed in osteoblasts, participates in the regulation of Wnt signaling ${ }^{35}$ and craniofacial malformations have been reported in Sfrp2 mutant mice ${ }^{36}$.

The 6 p21.1 region associated with nose bridge breadth extends across $\sim 500 \mathrm{~kb}$ overlapping the suppressor of Ty 3 homologue (S. cerevisiae; SUPT3H) gene and the $5^{\prime}$-half of the Runt-related transcription factor 2 (RUNX2) gene (Fig. 3d). Strongest association is seen for SNPs in the region of SUPT3H/RUNX2 overlap, peaking at SNP rs1852985 for both the ordinal and the quantitative assessment of nose bridge breadth (Fig. 3d, Supplementary Fig. 7). This region is known to contain key RUNX2 regulatory elements ${ }^{37}$ (Supplementary Fig. 12). Rare mutations in RUNX2 cause Cleidocranial dysplasia, an autosomal dominant disorder involving alterations of cranial ossification (OMIM \#119600). Runx2 has been shown to participate in the differentiation of mouse osteoblasts, chondrocyte and mesenchymal stem cells and bone development ${ }^{38}$, null Runx2 mutants showing a range of chondrocyte proliferation and maturation defects ${ }^{39}$. Interestingly, the length of a functional glutamine/alanine repeat in RUNX2 has been shown to correlate strongly with the evolution of facial length in dog breeds and, more broadly, in Carnivora ${ }^{40}$.

SNPs in the 7p13 region associated with nose wing breadth extend over $\sim 80 \mathrm{~kb}$ within the third intron of the GLI Family Zinc-Finger 3 gene (GLI3; Fig. 3e), a DNA-binding transcription factor. Strongest association for both the ordinal and quantitative assessments of nose wing breadth is observed for SNP rs17640804 (Tables 1 and 2, Fig. 3e, Supplementary Fig. 7), located in a genomic region with strong evolutionary conservation (Supplementary Fig. 13). Chromatin immunoprecipitation experiments have shown that rs17640804 can affect the binding of regulatory proteins ${ }^{41}$. GLI3 is known to act both as activator and repressor in the sonic hedgehog signalling pathway, a key regulatory of chondrocyte differentiation ${ }^{42}$. Interestingly, it has been shown experimentally that Gli3 interacts with Runx2 in the regulation of mouse osteoblast differentiation ${ }^{43}$. We therefore tested for statistical interaction between the GLI3 and RUNX2 index SNPs on nose bridge breadth and found it to be significant ( $P$ value $=0.004$, Supplementary Table 17), even though the GLI3 index SNP by itself does not have a significant effect on nose bridge breadth. Mutations in GLI3 have been shown to cause several Mendelian disorders associated with craniofacial and limb abnormalities, including GCPS (Greig cephalopolysyndactyly syndrome). GCPS is characterized by a range of craniofacial abnormalities including a broad nose ${ }^{44}$. A mouse null Gli3 mutant has been reported to show a range of craniofacial abnormalities, including a wider nose ${ }^{45}$.

Strongest association in 20p11 with the ordinal assessment of nose wing breadth was observed for SNP rs927833 located in LOC100270679, a long intergenic non-protein coding RNA (LINC01432). There is substantial LD around this SNP and suggestive evidence of association (that is, $P$ values $<10^{-5}$ ), for SNPs over a region of $\sim 400 \mathrm{~kb}$ extending to the Paired-box gene 1 (PAX1; Fig. 3f), a strong candidate gene in this region. PAX1 is a key developmental transcription factor which has been shown experimentally to affect chondrocyte differentiation through its participation in a regulatory pathway that also includes RUNX2 and SOX9 (ref. 46). More broadly, a Pax-Six-Eya-Dach (Dachshund) network, involving protein-protein and proteinDNA interactions impacting on a range of basic developmental processes has been described ${ }^{47}$. As indicated above, another $P A X$ gene $(P A X 3)$ has been twice reported to impact on nasion position $^{9,11}$, and we replicate that association here. A missense mutation in PAX1 has been shown to cause autosomal recessive oto-facio-cervical syndrome, a disorder characterized by various skeletal and facial abnormalities ${ }^{48}$. It has also been reported that mouse embryos with Gli3-null mutations display drastically 
reduced Pax1 expression, possibly mediated through Gli3's involvement in the sonic hedgehog signalling pathway ${ }^{49}$. Consistent with these experimental findings, we observe a significant statistical interaction of the GLI3 and PAX1 index SNPs on nose wing breadth $(P$ value $=0.005$, Supplementary Table 17).

\section{Discussion}

Since quantitative traits are expected to provide higher power for detecting genetic effects than categorical traits, most recent efforts to identify genes for facial features have focused on quantitative assessments from 3D image data $9,11,50,51$. However, thus far the use of these phenotyping tools has not resulted in many robust genetic finds, mainly the $P A X 3$-nasion position association replicated here ${ }^{9,11}$. Rather surprisingly, because of the comparatively lower power of non-quantitative phenotyping, we recently reported that using a simple ordinal phenotyping approach based on standard 2D photographs we were able to identify loci influencing pinna morphology ${ }^{12}$. Similar categorical rating scales have been used previously for the identification of genes for other anthropological features, such as pigmentation, hair type and tooth morphology ${ }^{25,52}$. Here, we confirm that categorical scales can be used to identify gene loci impacting on morphological features akin to those examined in certain anthropological and forensics settings ${ }^{6,53,54}$. We believe that our ability to detect genetic effects for such categorical traits arises from the high statistical power of the CANDELA sample for association testing of anthropological features, due to its comparatively large sample size, and particularly because of its extensive phenotypic and genetic diversity. This diversity relates to the admixed nature of this sample, admixture having involved continental populations with a relatively large genetic and phenotypic differentiation (mainly Europeans and Native Americans). This sample, thus, represents a sort of natural experiment facilitating the detection of genetic effects, especially for phenotypes differentiated between Europeans and Native Americans. Consistent with admixture having provided added power for association testing of the facial features examined, allele frequencies at the index SNPs in the novel face loci identified here show large differences between Europeans and East Asians/Native Americans and intermediate frequencies in the CANDELA sample (Table 3). Detection of genetic effects for these alleles would thus have relatively lower power in un-admixed continental populations. Consistent with this, independent evidence of association of chin shape with SNPs in the EDAR region has been recently reported in a Central Asian population with both Eastern and Western Eurasian ancestry ${ }^{21}$. Furthermore, power for the quantitative analyses performed here was likely increased by the preceding categorical analyses in that we focused on quantitative measures related to the ordinal traits, thus avoiding the considerable multiple-testing problem that can arise from the agnostic use of facial landmarks ${ }^{51}$.

Interestingly, we find no overlap between the gene regions affecting nose shape identified here and those we identified previously for pinna morphology in the same study sample ${ }^{12}$. Although, our current analyses certainly detect only some of the loci affecting these structures, our observations are consistent with the suggestion that facial features could be influenced by numerous genes with independent effects on different structures $^{22}$. In fact, anatomical studies have placed the nose and the pinna in different developmental modules ${ }^{55}$. The possibility that variation in specific craniofacial structures could result from the action of different genes might also contribute to explain why quantitative analyses using whole-face shape summaries from 3D images have had limited success in detecting significant genetic effects $9,11,50,51$. If different genes act mainly on different facial structures (and on specific aspects of those structures) higher power to detect these genetic effects could be provided by more narrowly defined variables (for example, distances) than by broad-shape summaries (for example, PCs).

Four of the gene regions identified here (DCHS2, RUNX2, GLI3 and $P A X 1)$ affect nose morphology. These results are consistent with the relatively high heritability of central middle face structures ${ }^{8}$ and the findings of the two published face-features GWAS, which also implicated mainly nose-related traits ${ }^{9,11}$. The shape of the human nose results from the coordinated development of mid-face bones and cartilages, including several in the nasal cavity ${ }^{56}$. Appropriately, the most compelling candidate genes in the regions we identified have wellestablished effects in cartilage and bone differentiation and have been shown to impact on craniofacial development in animal models. Interestingly, the analysis of genome sequences from modern and archaic humans (Neanderthals and Denisova) have identified DCHS2, GLI3 and RUNX2 among the top candidate genes harbouring highly differentiated variants and signatures of recent selection in the branches leading to these groups ${ }^{57,58}$. This observation has been interpreted as suggesting that these genes could be involved in the phenotypic differentiation of modern and archaic humans. Furthermore, using ancestry information and tests for accelerated evolution Claes et al. ${ }^{50}$ identified GLI3 as a gene undergoing rapid evolution in modern humans. The effect of $E D A R$ on chin protrusion adds to the developing picture of this gene having a multitude of phenotypic effects in populations with East Asian and Native American ancestry, the 370A allele having been associated so far with: increased sweat gland density ${ }^{27}$, straight hair ${ }^{12,26}$, lower beard and eye-brow thickness ${ }^{52}$, increased incisor shovelling ${ }^{25}$ and a range of pinna features ${ }^{12}$

In conclusion, we have identified five gene regions influencing normal variation in facial features. These regions harbour strong candidate genes, which independent evidence implicates in craniofacial development and evolution. It will be interesting to examine further the role that these gene regions might play in the evolutionary diversification of facial features in mammals, including the appearance of derived features in archaic and modern humans, as well as their potential involvement in the evolution of adaptive features of facial anatomy. The results presented here (and in related publications ${ }^{12,52}$ ) illustrate the high power provided by the CANDELA sample for the genetic analysis of phenotypes differentiated between Native Americans and Europeans. Further work on this sample, including additional quantitative trait analyses and the exploitation of $3 \mathrm{D}$ imaging techniques, should help delineate more fully the genetic architecture of the human face, including the possible overlap with gene regions implicated in common, complex alterations of facial development, such as NSCL/P.

\section{Methods}

Study subjects. In all, 6,275 volunteers from 5 countries (Colombia, $N=1,402$; Brasil, $N=658$; Chile, $N=1,760$; Mexico, $N=1,200$; and Peru, $N=1,255)$, part of the CANDELA consortium sample (http://www.ucl.ac.uk/silva/candela) ${ }^{13}$, aged between 18 and 45 years were included in this study (Supplementary Table 1). Ethics approval was obtained from: Universidad Nacional Autónoma de México (México), Universidad de Antioquia (Colombia), Universidad Perúana Cayetano Heredia (Perú), Universidad de Tarapacá (Chile), Universidade Federal do Rio Grande do Sul (Brasil) and University College London (UK). All participants provided written informed consent. Individuals with dysmorphologies, a history of facial surgery or trauma, or with BMI over 33 were excluded (due to the effect of obesity on facial features). Blood samples were collected by a certified phlebotomist and DNA extracted following standard laboratory procedures. Subsequent to the GWAS, an additional 501 individuals were recruited to serve as a replication sample (Supplementary Fig. 4). These individuals were recruited following the same procedures as for the sample included in the GWAS.

Ordinal phenotyping. This was carried out in the same way for the GWAS and replication samples. Right side and frontal photographs were used to score 14 facial 
traits. This included: chin shape and protrusion, cheekbone and brow-ridge protrusion, forehead profile, upper and lower lip thickness and seven nose features (breadth of nasal root, bridge and wing, columella inclination, nose protrusion, nose profile and nose tip shape). These features were selected based on their reported variation in European populations ${ }^{5}$. Software to assist scoring of photographs was developed in MATLAB (ref. 59). Intraclass correlation coefficients (ICCs) ${ }^{60}$ calculated by repeated scoring of photographs of 450 subjects by two independent raters (M.F.-G. and I.P.A.) indicate a moderate-to-high intrarater reliability of the trait scores (Supplementary Table 3), with relatively lower inter-rater reliability for certain traits. Photographs for all the volunteers were scored by the same rater (M.F.-G.).

Quantitative phenotyping. Quantitative phenotypes were obtained using Procrustes-adjusted 3D facial landmark coordinates available for 2,955 of the individuals included in the ordinal trait GWAS. These coordinates were obtained for 34 anatomical landmarks as detailed in ref. 20 (Supplementary Fig. 5). Briefly, landmarks were placed and raw 3D coordinates obtained using Photomodeler software and five facial photographs (taken at $0^{\circ}, 45^{\circ}, 90^{\circ}, 135^{\circ}$ and $180^{\circ}$, where $0^{\circ}$ is the left side view). The raw 3D landmark coordinates were Procrustes-adjusted using the MorphoJ software ${ }^{61}$. Quantitative measurements (distances and angles) were defined corresponding to seven of the ordinal traits initially examined (Supplementary Table 9). Since no 3D landmarks allowing quantitative proxies for nose root and bridge breadth were available we placed 2D landmarks on the frontal photographs of the same individuals with 3D landmarks (Supplementary Fig. 5, Supplementary Table 10): two landmarks were added each for nasal root and for nose bridge width, in addition to the major frontally visible 3D landmarks. Since the $3 \mathrm{D}$ coordinates are free of head tilts and rotations (thus allowing more accurate measurements) the $2 \mathrm{D}$ coordinates were calibrated with reference to the $3 \mathrm{D}$ coordinates using corresponding frontal landmarks (having both $2 \mathrm{D}$ and $3 \mathrm{D}$ coordinates) (Supplementary Fig. 5a,b).

DNA genotyping and quality control. DNA samples from participants were genotyped on the Illumina HumanOmniExpress chip including 730,525 SNPs. PLINK v1.9 (ref. 62) was used to exclude SNPs and individuals with $>5 \%$ missing data, markers with minor-allele frequency $<1 \%$, related individuals (Plink IBD estimate $>0.1$ ), and those who failed the X-chromosome sex concordance check (sex estimated from X-chromosome heterozygosity not matching recorded sex information). After applying these filters 671,038 SNPs and 5,958 individuals (1,303 from Colombia, 608 from Brasil, 1,651 from Chile, 1,165 from Mexico, 1,231 from Peru) were retained for further analysis. Due to the admixed nature of the study sample (Supplementary Fig. 2) there is an inflation in Hardy-Weinberg $P$ values. We therefore did not exclude markers based on Hardy-Weinberg deviation, but performed stringent quality controls at software and biological levels, and checked the genotyping cluster plots for each index SNP manually (Supplementary Fig. 14). The replication sample was genotyped in the same way and the genotype data submitted to the same quality controls as for the GWAS sample.

SNP genotype imputation. The chip genotype data was phased using SHAPEIT2 (ref. 63). IMPUTE2 (ref. 64) was then used to impute genotypes at untyped SNPs using variant positions from the 1000 Genomes Phase I data. The 1000 Genomes reference data set includes haplotype information for 1,092 individuals across the world for $36,820,992$ variant positions. Positions that are monomorphic in 1000 Genomes Latin American samples (CLM, MXL and PUR) were excluded, leading to $11,025,002$ SNPs being imputed in our data set. Of these, 48,695 had imputation quality scores $<0.4$ and were excluded. Chip genotyped SNPs having a low concordance value $(<0.7)$ or a large gap between info and concordance values (info_type0-concord_type $0>0.1$ ), which might be indicators of poor genotyping, were also removed, both from the imputed and chip data set. The IMPUTE2 genotype probabilities at each locus were converted into best-guess genotypes using PLINK $^{62}$ (at the default setting of $<0.1$ uncertainty). SNPs with proportion of samples with uncalled genotypes $>5 \%$ and minor-allele frequency $<1 \%$ were excluded. The final imputed data set contained genotypes for 9,117,642 SNPs.

Statistical genetic analyses. Narrow-sense heritability (defined as the additive phenotypic variance explained by a Genetic Relatedness Matrix, GRM, computed from the SNP data) was estimated using GCTA ${ }^{15}$ by fitting an additive linear model with a random-effect term whose variance is given by the GRM, with age, sex and BMI as covariates. The GRM was obtained using the LDAK approach ${ }^{14}$, which accounts for LD between SNPs. An LD-pruned set of 93,328 autosomal SNPs was used to estimate European, African and Native American ancestry using supervised runs of ADMIXTURE ${ }^{65}$ (Supplementary Fig. 2). Reference parental populations included in the ADMIXTURE analyses consisted of Africans and Europeans from HAPMAP and selected Native Americans, as described in Ruiz-Linares et al. ${ }^{13}$

PLINK 1.9 (ref. 62) was used to perform the primary genome-wide association tests for each phenotype using multiple linear regression with an additive genetic model incorporating age, sex, BMI and five genetic PCs as covariates. Association analyses were performed on the imputed data set with two approaches: using the best-guess imputed genotypes in PLINK and using the IMPUTE2 genotype probabilities in SNPTEST v2.5 (ref. 66). Both were consistent with each other and with the results from the chip genotype data. For analysis of the $\mathrm{X}$ chromosome an inactivation model was used (male genotypes encoded as $0 / 2$ and female genotypes as 0/1/2). The genetic PCs were obtained (using PLINK 1.9 (ref. 62) from an LD-pruned dataset of 93,328 SNPs. They were selected by inspecting the proportion of variance explained and checking scree and PC scatter plots (Supplementary Fig. 3a). Individual outliers were removed and PCs recalculated after each removal. The top PCs appear to be a good proxy for continental ancestry (Supplementary Fig. 3b). Using these PCs the Q-Q plots (Supplementary Fig. 3c) for all association tests showed no sign of inflation, the genomic control factor lambda being $<1.02$ in all cases (Supplementary Fig. 3d), thus confirming that we are appropriately accounting for population stratification ${ }^{67}$. Similar analyses were applied for association testing of the index SNPs followed-up in the replication sample. To account for multiple testing we also applied a global false-discovery rate test using the Benjamini-Hochberg procedure across all traits and SNPs (Supplementary Table 6). To account for the correlations between traits, a multivariate GWAS was also performed, testing for association with all facial traits simultaneously using a Wald test conditioned on all covariates (Supplementary Table 8). A meta-analysis was carried out for the index SNPs identified in the primary analyses by testing for association separately in each country sample and combining the results (using the PLINK implementation of the meta-analysis software METAL ${ }^{18}$ ). Forest plots were produced with MATLAB. Cochran's Q-statistic was computed for each trait to test for effect-size heterogeneity across country samples. The fraction of trait variance explained by the covariates, by each index SNP, and by all index SNPs altogether, were estimated from linear regression models implemented using $\mathrm{R}^{2}$ (Supplementary Table 14). To evaluate the role of NSCL/P loci on the facial traits examined we selected index SNPs in the 15 associated regions reported in the literature (Supplementary Table 15) and performed individual SNP associations, global Kolmogorov-Smirnov tests and Polygenic Risk Score tests using PLINK.

Mouse analyses. Animal studies were reviewed and approved by The Roslin Institute Animal Welfare and Ethical Review Body (AWERB). The humane care and use of mice (Mus musculus) in this study was carried out under the authority of the appropriate UK Home Office Project License. The mouse samples and head photographs examined are from the same set described fully in Adhikari et al. ${ }^{12}$ Briefly, we included fourteen and 15-day-old animals (17 males and 23 female). The mouse genotypes were Edar ${ }^{d l J}$ (a loss of function EDARp.E379K mutation ${ }^{32}$ ) as either homozygote or heterozygote, wild-type $(+/+)$ and the homozygous Edar ${ }^{T g 951}$ line (which has $\sim 16$ extra copies of Edar per haploid genome ${ }^{31}$ ). Thirteen 2D anatomical landmarks were placed on lateral photographs of the mouse heads, using TPSDig and TPSUtil (http://life.bio.sunysb.edu/morph/; Supplementary Fig. 8). Generalized procrustes analysis was carried out using the software Morphoj ${ }^{61}$ to check whether the distribution of landmarks was homogeneous. No outliers were detected. Mouse mandible length was measured using the landmark coordinates (as detailed in Supplementary Figs 8 and 9) and mandible length (as a proportion of head size, measured directly on the heads) was regressed onto age, sex and Edar genotype. In this regression Edar genotype was coded as 1-4 based on increasing Edar expression: 1-Edar ${ }^{\text {dll/dll }}$ homozygotes, $2-E d a r^{d l J /}+$ heterozygotes, 3-wild-type ${ }^{+/+}$mice and 4-Edar ${ }^{T g 951 / T g 951}$ homozygotes (Supplementary Table 16).

\section{References}

1. Reyes-Centeno, H., Hubbe, M., Hanihara, T., Stringer, C. \& Harvati, K. Testing modern human out-of-Africa dispersal models and implications for modern human origins. J. Hum. Evol. 87, 95-106 (2015).

2. Harvati, K. \& Weaver, T. D. Human cranial anatomy and the differential preservation of population history and climate signatures. Anat. Rec. A Discov. Mol. Cell. Evol. Biol. 288, 1225-1233 (2006).

3. von Cramon-Taubadel, N. Evolutionary insights into global patterns of human cranial diversity: population history, climatic and dietary effects. J. Anthropol. Sci. 92, 43-77 (2014).

4. Sheehan, M. J. \& Nachman, M. W. Morphological and population genomic evidence that human faces have evolved to signal individual identity. Nat. Commun. 5, 4800 (2014).

5. Ritz-Timme, S. et al. A new atlas for the evaluation of facial features: advantages, limits, and applicability. Int. J. Legal. Med. 125, 301-306 (2011).

6. Dirkmaat, D. A companion to forensic anthropology, xxxvi 716 (Wiley-Blackwell, 2012).

7. Martinez-Abadias, N. et al. Heritability of human cranial dimensions: comparing the evolvability of different cranial regions. J. Anat. 214, 19-35 (2009).

8. Weinberg, S. M., Parsons, T. E., Marazita, M. L. \& Maher, B. S. Heritability of face shape in twins: a preliminary study using 3D stereophotogrammetry and geometric morphometrics. Dent. 3000, 1 (2013).

9. Liu, F. et al. A genome-wide association study identifies five loci influencing facial morphology in Europeans. PLoS Genet. 8, e1002932 (2012).

10. Twigg, S. R. \& Wilkie, A. O. New insights into craniofacial malformations. Hum. Mol. Genet. 24, R50-R59 (2015). 
11. Paternoster, L. et al. Genome-wide association study of three-dimensional facial morphology identifies a variant in PAX3 associated with nasion position. Am. J. Hum. Genet. 90, 478-485 (2012).

12. Adhikari, K. et al. A genome-wide association study identifies multiple loci for variation in human ear morphology. Nat. Commun. 6, 7500 (2015).

13. Ruiz-Linares, A. et al. Admixture in Latin America: geographic structure, phenotypic diversity and self-perception of ancestry based on 7,342 individuals. PLoS Genet. 10, e1004572 (2014).

14. Speed, D., Hemani, G., Johnson, M. R. \& Balding, D. J. Improved heritability estimation from genome-wide SNPs. Am. J. Hum. Genet. 91, 1011-1021 (2012)

15. Yang, J., Lee, S. H., Goddard, M. E. \& Visscher, P. M. GCTA: a tool for genome-wide complex trait analysis. Am. J. Hum. Genet. 88, 76-82 (2011)

16. Carson, E. A. Maximum likelihood estimation of human craniometric heritabilities. Am. J. Phys. Anthropol. 131, 169-180 (2006).

17. Purcell, S. et al. PLINK: a tool set for whole-genome association and population-based linkage analyses. Am. J. Hum. Genet. 81, 559-575 (2007).

18. Willer, C. J., Li, Y. \& Abecasis, G. R. METAL: fast and efficient meta-analysis of genomewide association scans. Bioinformatics. 26, 2190-2191 (2010).

19. O'Reilly, P. F. et al. MultiPhen: joint model of multiple phenotypes can increase discovery in GWAS. PLoS ONE 7, e34861 (2012).

20. Quinto-Sanchez, M. et al. Facial asymmetry and genetic ancestry in Latin American admixed populations. Am. J. Phys. Anthropol. 157, 58-70 (2015).

21. Peng, Q. et al. EDARV370A associated facial characteristics in Uyghur population revealing further pleiotropic effects. Hum. Genet. 135, 99-108 (2015).

22. Boehringer, S. et al. Genetic determination of human facial morphology: links between cleft-lips and normal variation. Eur. J. Hum. Genet. 19, 1192-1197 (2011).

23. Sadier, A., Viriot, L., Pantalacci, S. \& Laudet, V. The ectodysplasin pathway: from diseases to adaptations. Trends Genet. 30, 24-31 (2014).

24. Bryk, J. et al. Positive selection in East Asians for an EDAR allele that enhances NF-kappaB activation. PLoS ONE 3, e2209 (2008).

25. Park, J. H. et al. Effects of an Asian-specific nonsynonymous EDAR variant on multiple dental traits. J. Hum. Genet. 57, 508-514 (2012).

26. Tan, J. et al. The adaptive variant EDARV370A is associated with straight hair in East Asians. Hum. Genet. 132, 1187-1191 (2013).

27. Kamberov, Y. G. et al. Modeling recent human evolution in mice by expression of a selected EDAR variant. Cell 152, 691-702 (2013).

28. Cluzeau, C. et al. Only four genes (EDA1, EDAR, EDARADD, and WNT10A) account for $90 \%$ of hypohidrotic/anhidrotic ectodermal dysplasia cases. Hum. Mutat. 32, 70-72 (2011).

29. Goodwin, A. F. et al. Craniofacial morphometric analysis of individuals with X-linked hypohidrotic ectodermal dysplasia. Mol. Genet. Genomic Med. 2, 422-429 (2014)

30. Headon, D. J. et al. Gene defect in ectodermal dysplasia implicates a death domain adapter in development. Nature 414, 913-916 (2001).

31. Mou, C. et al. Enhanced ectodysplasin-A receptor (EDAR) signaling alters multiple fiber characteristics to produce the East Asian hair form. Hum. Mutat. 29, 1405-1411 (2008).

32. Monreal, A. W. et al. Mutations in the human homologue of mouse $\mathrm{dl}$ cause autosomal recessive and dominant hypohidrotic ectodermal dysplasia. Nat. Genet. 22, 366-369 (1999)

33. Bornert, F. et al. Subtle morphological changes in the mandible of Tabby mice revealed by micro-CT imaging and elliptical Fourier quantification. Front. Physiol. 2, 15 (2011).

34. Le Pabic, P., Ng, C. \& Schilling, T. F. Fat-Dachsous signaling coordinates cartilage differentiation and polarity during craniofacial development. PLoS Genet. 10, e1004726 (2014).

35. Nakajima, H. et al. Wnt modulators, SFRP-1, and SFRP-2 are expressed in osteoblasts and differentially regulate hematopoietic stem cells. Biochem. Biophys. Res. Commun. 390, 65-70 (2009).

36. Kurosaka, H., Iulianella, A., Williams, T. \& Trainor, P. A. Disrupting hedgehog and WNT signaling interactions promotes cleft lip pathogenesis. J. Clin. Invest. 124, 1660-1671 (2014).

37. Napierala, D. et al. Mutations and promoter SNPs in RUNX2, a transcriptional regulator of bone formation. Mol. Genet. Metab. 86, 257-268 (2005).

38. Fujita, T. et al. Runx2 induces osteoblast and chondrocyte differentiation and enhances their migration by coupling with PI3K-Akt signaling. J. Cell Biol. 166, 85-95 (2004).

39. Yoshida, C. A. et al. Runx 2 and Runx 3 are essential for chondrocyte maturation, and Runx2 regulates limb growth through induction of Indian hedgehog. Genes Dev. 18, 952-963 (2004).

40. Sears, K. E., Goswami, A., Flynn, J. J. \& Niswander, L. A. The correlated evolution of Runx 2 tandem repeats, transcriptional activity, and facial length in carnivora. Evol. Dev. 9, 555-565 (2007).

41. Feng, J. et al. A genome-wide survey over the ChIP-on-chip identified androgen receptor-binding genomic regions identifies a novel prostate cancer susceptibility locus at 12q13.13. Cancer Epidemiol. Biomarkers Prev. 20, 2396-2403 (2011).

42. Pan, A., Chang, L., Nguyen, A. \& James, A. W. A review of hedgehog signaling in cranial bone development. Front. Physiol. 4, 61 (2013).

43. Rice, D. P. et al. Gli3Xt-J/Xt-J mice exhibit lambdoid suture craniosynostosis which results from altered osteoprogenitor proliferation and differentiation. Hum. Mol. Genet. 19, 3457-3467 (2010).

44. Vortkamp, A., Gessler, M. \& Grzeschik, K. H. GLI3 zinc-finger gene interrupted by translocations in Greig syndrome families. Nature 352, 539-540 (1991).

45. Hui, C. C. \& Joyner, A. L. A mouse model of greig cephalopolysyndactyly syndrome: the extra-toes mutation contains an intragenic deletion of the Gli3 gene. Nat. Genet. 3, 241-246 (1993).

46. Takimoto, A., Mohri, H., Kokubu, C., Hiraki, Y. \& Shukunami, C. Pax1 acts as a negative regulator of chondrocyte maturation. Exp. Cell. Res. 319, 3128-3139 (2013).

47. Wagner, G. P. The developmental genetics of homology. Nat. Rev. Genet. 8, 473-479 (2007).

48. Pohl, E. et al. A hypofunctional PAX1 mutation causes autosomal recessively inherited otofaciocervical syndrome. Hum. Genet. 132, 1311-1320 (2013).

49. Buttitta, L., Mo, R., Hui, C. C. \& Fan, C. M. Interplays of Gli2 and Gli3 and their requirement in mediating Shh-dependent sclerotome induction. Development 130, 6233-6243 (2003).

50. Claes, P. et al. Modeling 3D facial shape from DNA. PLoS Genet. 10, e1004224 (2014).

51. Peng, S. et al. Detecting genetic association of common human facial morphological variation using high density $3 \mathrm{D}$ image registration. PLoS. Comput. Biol. 9, e1003375 (2013).

52. Adhikari, K. et al. The genetic basis of variation in facial and scalp hair: a genome-wide association study in admixed Latin Americans. Nat. Commun. doi:10.1038/ncomms10815 (2016).

53. Ritz-Timme, S. et al. Metric and morphological assessment of facial features: a study on three European populations. Forensic Sci. Int. 207, 239 e1-8 (2011)

54. Hefner, J. T. \& Ousley, S. D. Statistical classification methods for estimating ancestry using morphoscopic traits. J. Forensic Sci. 59, 883-890 (2014).

55. Esteve-Altava, B., Diogo, R., Smith, C., Boughner, J. C. \& Rasskin-Gutman, D. Anatomical networks reveal the musculoskeletal modularity of the human head. Sci. Rep. 5, 8298 (2015).

56. Lieberman, D. The evolution of the human head, xi 756 (Belknap Press of Harvard University Press, 2011).

57. Green, R. E. et al. A draft sequence of the Neandertal genome. Science 328, 710-722 (2010).

58. Prufer, K. et al. The complete genome sequence of a Neanderthal from the Altai Mountains. Nature 505, 43-49 (2014).

59. The MathWorks, I. MATLAB and Statistics Toolbox Release 2013b (Natick, 2013)

60. Shrout, P. E. \& Fleiss, J. L. Intraclass correlations: uses in assessing rater reliability. Psychol. Bull. 86, 420-428 (1979).

61. Klingenberg, C. P. MorphoJ: an integrated software package for geometric morphometrics. Mol. Ecol. Resour. 11, 353-357 (2011).

62. Chang, C. C. et al. Second-generation PLINK: rising to the challenge of larger and richer datasets. Gigascience 4, 7 (2015).

63. O'Connell, J. et al. A general approach for haplotype phasing across the full spectrum of relatedness. PLoS Genet. 10, e1004234 (2014).

64. Howie, B., Fuchsberger, C., Stephens, M., Marchini, J. \& Abecasis, G. R. Fast and accurate genotype imputation in genome-wide association studies through pre-phasing. Nat. Genet. 44, 955-959 (2012).

65. Alexander, D. H., Novembre, J. \& Lange, K. Fast model-based estimation of ancestry in unrelated individuals. Genome Res. 19, 1655-1664 (2009).

66. Marchini, J. \& Howie, B. Genotype imputation for genome-wide association studies. Nat. Rev. Genet. 11, 499-511 (2010).

67. Patterson, N., Price, A. L. \& Reich, D. Population structure and eigenanalysis. PLoS Genet. 2, 2074-2093 (2006).

68. Pruim, R. J. et al. LocusZoom: regional visualization of genome-wide association scan results. Bioinformatics 26, 2336-2337 (2010).

69. Barrett, J. C., Fry, B., Maller, J. \& Daly, M. J. Haploview: analysis and visualization of LD and haplotype maps. Bioinformatics 21, 263-265 (2005).

70. Reich, D. et al. Reconstructing native American population history. Nature 488, 370-374 (2012).

\section{Acknowledgements}

We thank the volunteers for their enthusiastic support for this research. We also thank Esra Konca, Ivan Pulgar-Alarcón, Alvaro Alvarado, Mónica Ballesteros Romero, Ricardo Cebrecos, Miguel Ángel Contreras Sieck, Francisco de Ávila Becerril, Joyce De la Piedra María Teresa Del Solar, Paola Everardo Martínez, William Flores, Martha Granados Riveros, Rosilene Paim, Ricardo Gunski, Sergeant João Felisberto Menezes Cavalheiro, Major Eugênio Correa de Souza Junior, Wendy Hart, Ilich Jafet Moreno, Paola León-Mimila, Francisco Quispealaya, Diana Rogel Diaz, Ruth Rojas, Norman Russell and Vanessa Sarabia, for assistance with volunteer recruitment, sample processing and data 
entry. We are indebted to Emiliano Bellini (www.belliniplastica.blogspot.com) for the illustration in Fig. 1, and to Elizabeth Guajardo Celsi for some of the face drawings used in the phenotyping software. We thank three anonymous reviewers for thoughtful comments that significantly improved this paper. We are very grateful to the institutions that kindly provided facilities for the assessment of volunteers, including: Escuela Nacional de Antropología e Historia and Universidad Nacional Autónoma de México (México); Universidade Federal do Rio Grande do Sul (Brasil); $13^{\circ}$ Companhia de Comunicações Mecanizada do Exército Brasileiro (Brasil); Pontificia Universidad Católica del Perú, Universidad de Lima and Universidad Nacional Mayor de San Marcos (Perú). This work was funded by grants from the Leverhulme Trust (F/07 134/DF to A.R.-L), BBSRC (BB/I021213/1 to A.R.-L), Universidad de Antioquia (CODI sostenibilidad de grupos 2013-2014 and MASO 2013-2014), Conselho Nacional de Desenvolvimento Científico e Tecnológico, Fundação de Amparo à Pesquisa do Estado do Rio Grande do Sul (Apoio a Núcleos de Excelência Program) and Fundação de Aperfeiçoamento de Pessoal de Nível Superior.

\section{Author contributions}

Conceived and designed study: K.A., M.F.-G., J.R., R.G.-J., D.H., D.B., A.R.-L. Contributed reagents/material: M.F.-G., J.M.-R., V.A.A., C.J., W.A., R.B.L., G.M.P., J.G.V., H.V.-R., T.H., V.R., C.C.S.d.C., M.H., V.V., V.G., D.H. Performed experiments: M.F.G., J.M.-R., J.C.C.-D., V.A.A., M.Q.-S., D.H. Analysed data: KA, M.F.-G., M.Q.-S., D.H., A.R.-L. Supervised research (PI): J.G.-V., C.G., G.P., L.S.-F., F.M.S., M.-C.B., S.C.-Q., F.R., G.B., R.G.-J., D.H., D.B., A.R.-L. Wrote the manuscript, incorporating input from other authors: K.A., M.F.-G., A.R.-L. Critical revision of the manuscript: R.G.-J., D.H., D.B.

\section{Additional information}

Accession codes: The MATLAB program used to perform the ordinal scoring of facial features can be downloaded from http://www.ucl.ac.uk/silva/candela. $P$ values for all SNPs tested in the GWAS analyses will be hosted in GWAS Central (http:// www.gwascentral.org/), and also made available through http://www.ucl.ac.uk/silva/ candela on the next data release of the GWAS Central database, scheduled for June 2016.

Supplementary Information accompanies this paper at http://www.nature.com/ naturecommunications

Competing financial interests: The authors declare no competing financial interests.

Reprints and permission information is available online at http://npg.nature.com/ reprintsandpermissions/

How to cite this article: Adhikari, K. et al. A genome-wide association scan implicates DCHS2, RUNX2, GLI3, PAX1 and EDAR in human facial variation. Nat. Commun. 7:11616 doi: $10.1038 /$ ncomms11616 (2016).

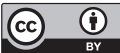

This work is licensed under a Creative Commons Attribution 4.0 International License. The images or other third party material in this article are included in the article's Creative Commons license, unless indicated otherwise in the credit line; if the material is not included under the Creative Commons license, users will need to obtain permission from the license holder to reproduce the material. To view a copy of this license, visit http://creativecommons.org/licenses/by/4.0/ 